\title{
Thermal rectification and negative differential thermal resistance in lattices with mass gradient
}

\author{
Nuo Yang, ${ }^{1}$ Nianbei Li, ${ }^{1}$ Lei Wang, ${ }^{1}$ and Baowen $\mathrm{Li}^{1,2,},{ }^{1}$ \\ ${ }^{1}$ Department of Physics and Centre for Computational Science and Engineering, National University of Singapore, 117542 Singapore \\ ${ }^{2}$ NUS Graduate School for Integrative Sciences and Engineering, 117597 Singapore \\ (Dated: 19 January 2007, Accepted for publication in Phys. Rev. B as Rapid Com.)
}

\begin{abstract}
We study thermal properties of one dimensional(1D) harmonic and anharmonic lattices with mass gradient. It is found that the temperature gradient can be built up in the 1D harmonic lattice with mass gradient due to the existence of gradons. The heat flow is asymmetric in the anharmonic lattices with mass gradient. Moreover, in a certain temperature region the negative differential thermal resistance is observed. Possible applications in constructing thermal rectifier and thermal transistor by using the graded material are discussed.
\end{abstract}

PACS numbers: $65.09+\mathrm{i}, 65.60+\mathrm{a}, 66.70+\mathrm{f}, 63.20 \mathrm{Ry}$

Theoretical studies of heat conduction in low dimensional nonlinear lattices in past years have not only enriched our understanding of the microscopic physical mechanism of heat conduction, but also suggested some useful thermal device models such as rectifier/diode [1] and thermal transistor[2] for controlling heat flow. More importantly, a two segment thermal rectifier has been realized experimentally by using nanotubes[3], which implies that we can control and manipulate phonons like we do for electrons.

On the other hand, the functional graded materials (FGM) have attracted increasing attention in many fields ranging from aerospace, electronics, optics and bio-materials etc. due to the intriguing properties [4, 5]. The FGM are a kind of inhomogeneous materials whose compositions and/or structures change gradually in space which results in corresponding changes in physical properties such as electric, mechanical, thermal, and optical properties. The FGM are abundant in nature, and can be also purposely manufactured [4, 6].

However, compared with optical, mechanical and many other properties, the thermal properties of the graded materials have not yet been fully studied (see recent review article [5] and the references therein).

In this paper, we study thermal properties of the FGM represented by 1D harmonic and anharmonic lattices with mass gradient. For convenience, we call the lattices mass graded harmonic/anharmonic lattices. They can be used to model superlattice or layered structures. As it will be seen that the $1 \mathrm{~d}$ graded lattices exhibit new physics such as asymmetric heat flow and negative differential thermal resistance, two essential properties used to construct thermal rectifier and thermal transistor. Therefore, the graded material might find new applications in heat controlling and management.

We consider a 1D mass graded chain, which is equivalent to a chain with graded coupling constants[7]. Fig 1 a) shows the configuration. The mass of the $i^{\text {th }}$ particle is $M_{i}=M_{M A X}-$ $(i-1)\left(M_{M A X}-M_{M I N}\right) /(N-1)$, where $M_{M A X}$ is the mass of the particle at the left end and $M_{M I N}$ is that of the particle at the right end. $N$ is the total number of the particles. The Hamiltonian of this model is:

$$
H=\sum_{i}\left[\frac{p_{i}^{2}}{2 M_{i}}+V\left(x_{i}-x_{i-1}\right)\right]
$$

here $x_{i}$ is the position of the $i^{\text {th }}$ particle. Without loss of generality, $V$ takes an anharmonic form, namely, $\left(x_{i}-x_{i-1}-a\right)^{2} / 2+$ $\beta\left(x_{i}-x_{i-1}-a\right)^{4} / 4$, which is a Fermi-Pasta-Ulam (FPU) $\beta$ potential. So the system is called a graded FPU lattice. The lattice constant $a=1.0$ and the coupling constant $\beta=1.0$. In the case of $\beta=0$, this lattice reduces to a graded harmonic chain.

In our simulations we use both fixed and free boundary conditions. The stochastic heat baths are put on the $1^{s t}, 2^{\text {nd }}$, $(N-1)^{t h}$ and $N^{\text {th }}$ particles with temperature $T_{L}$ and $T_{R}$ respectively. The equations of motion (EOM) of these four particles are described by the Langevin equations:

$M_{i} \ddot{x}_{i}=F\left(x_{i}-x_{i-1}\right)-F\left(x_{i+1}-x_{i}\right)- \begin{cases}\xi_{L}-\lambda_{L} \dot{x}_{i}, & i=1,2 \\ \xi_{R}-\lambda_{R} \dot{x}_{i}, & i=N-1, N,\end{cases}$

where $\xi_{L / R}$ are independent Wiener processes with zero mean, variance $2 \lambda_{L / R} k_{B} T_{L / R}$ and force $F=-\partial V / \partial x$. To minimize the Kapitza resistance between the bath particle and its neighbor, we set $\lambda_{L / R} / M_{i}=0.1$. Both the fourth-order Runge-Kutta and the velocity Verlet algorithm are used to integrate the EOM. Differences between results of these two integration methods are negligible. Simulations are performed long enough ( $>10^{7}$ time units) such that the system reaches a stationary state where the local heat flux is constant along the chain.

We start with the mass graded harmonic lattice, i.e. $\beta=0$ in the potential $V(x)$. Fig 1 b) shows the temperature profile with different lattice length $N$ and different boundary conditions. $M_{M A X}$ and $M_{M I N}$ are fixed at 10 and 1, respectively. It is well known that no temperature gradient can be built up along a homogenous harmonic lattice[8]. However, in the mass graded harmonic lattice, temperature gradient is clearly seen.

The local flux at site $i$ is defined as

$$
J_{i}=\frac{1}{2} a\left\langle\left(\dot{x}_{i+1}+\dot{x}_{i}\right) F\left(x_{i+1}-x_{i}\right)\right\rangle .
$$

After the system reaches a stationary state, $J_{i}$ is independent of site position $i$, so the flux can be denoted as $J$. The heat conductivity is calculated by $\kappa=-J /(d T / d x)$. We should stress that in calculating the temperature gradient, we get rid of temperature jumps at the two boundaries. In Fig 1(c), the heat conductivity versus $N$ for different boundary conditions 

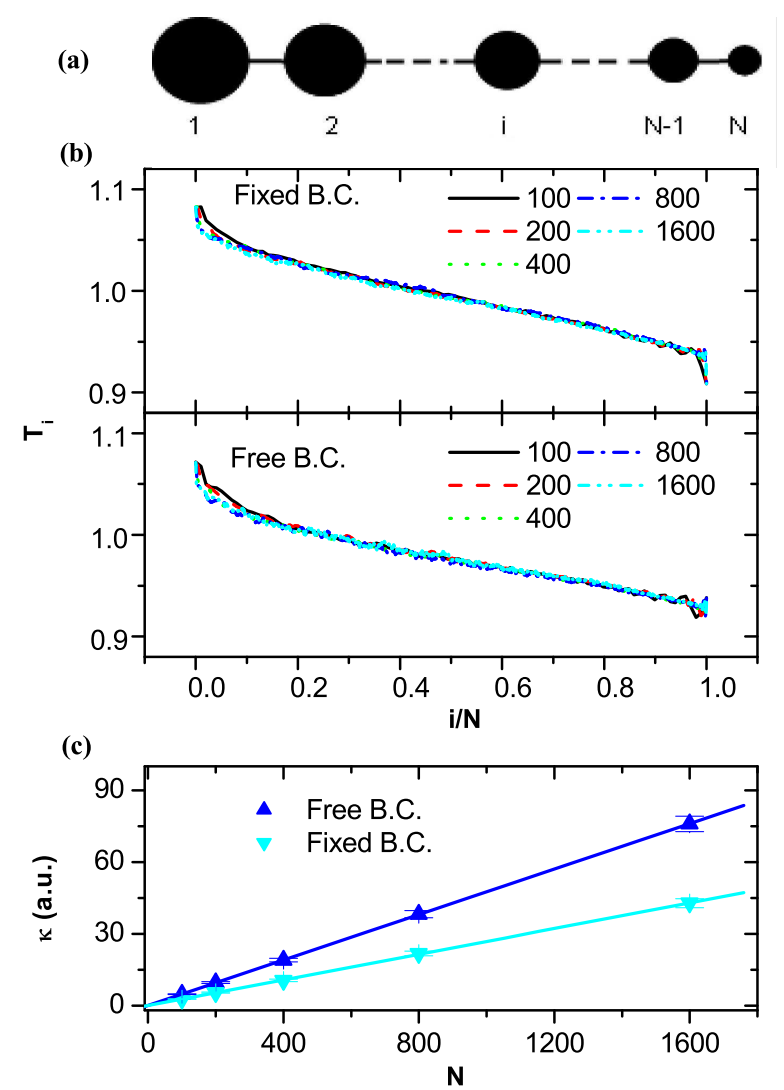

FIG. 1: (Color on-line) (a) Schematic picture of the mass graded harmonic lattice. (b) Temperature profile for the lattice with fixed and free boundary conditions. (c) The heat conductivity $\kappa$ versus system size $N$ for the lattice with $M_{M A X}=10$ and $M_{M I N}=1 . \kappa=A N$, where $A=0.048$ and 0.027 for free and fixed boundary conditions, respectively. The temperatures of two baths are $T_{L}=1.1$ and $T_{R}=$ 0.9 for left and right end, respectively.

are plotted. For the same lattice length $N$, heat conductivities with fixed boundaries are lower than those with free boundaries, because there is a limitation that all vibrational eigenmodes must vanish at boundaries in fixed boundary cases. It is clearly seen that the heat conductivity diverges linearly with length $N$. This linear property is independent of boundary condition. It is different from that of disordered harmonic lattice where the thermal conductivity diverges with system size as $\sqrt{N}$ and $1 / \sqrt{N}$ for free and fixed boundary condition, respectively[9].

The linear divergence of thermal conductivity on system size is a new phenomenon. As we know that in 1D homogenous harmonic lattices, no temperature gradient can be built up and thus thermal conductivity cannot be defined[10]. In order to understand the underlying mechanism, we have to invoke the vibrational eigenmodes of the graded system. It is found in Ref.[7] that there is a critical frequency $\omega_{c}$, which is the maximum eigenfrequency of the corresponding homogeneous harmonic lattice with $M_{i}=M_{M A X}$, where $M_{M A X}$ is the maximal mass in the graded harmonic lattice. The modes with $\omega<\omega_{c}$ can be well extended over the whole chain, whereas

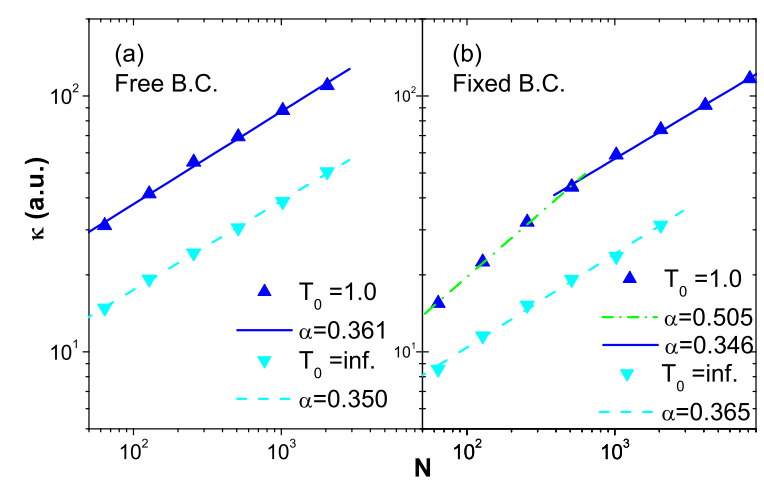

FIG. 2: (Color on-line) The heat conductivity $\kappa$ versus system size $N$ for graded FPU lattice under free boundary conditions (a) and fixed boundary conditions (b) with $M_{M A X}=10$ and $M_{M I N}=1$. In all cases, $\kappa \propto N^{\alpha}$. The values of $\alpha$ are given in the figure.

those with $\omega>\omega_{c}$ ( called gradons) are localized at the side with lighter masses. Therefore, the formation of temperature gradient in the graded harmonic lattice is attributed to the localization of the gradons.

In the following, we focus on the mass graded anharmonic lattice. We first examine the size effect of heat conductivity and its dependence on temperature. It is found that the thermal conductivity diverges with system size as, $\kappa \sim N^{\alpha}$. Fig 2 (a) shows the temperature effect of the divergent exponent under free boundary condition. The value of $\alpha$ does not change very much, it almost keeps the same value of 0.36 (0.35) when the average temperature $T_{0}$ is increased from 1.0 to infinity. The value of the divergence exponent is very close to the results from the renormalization group for $1 \mathrm{~d}$ hydrodynamic systems [11], and that one for the hard core model[12]. The calculation for infinite $T_{0}$ is actually realized by discarding the quadratic term (of potential) in Hamiltonian since this term is negligible compared with the quartic term in infinite temperature limit.

Fig (b) shows the temperature effect of the divergent exponent under fixed boundary conditions. In the case of $T_{0}=1$, the best fitting with all available date up to $N=512$ gives rise to an $\alpha=0.51$. However, the best fitting for values of the largest five $N(512 \leqslant N \leqslant 8192)$ gives rise to $\alpha=0.35$ which is very close to the value at the infinite temperature. The $\alpha=0.51$ might be a finite size effect.

The value of $\alpha$ for free boundary condition is quite close to the value for fixed boundary condition. Therefore, this result seems to be very similar to the homogenous FPU- $\beta$ lattice, namely, the divergent exponent $\alpha$ seems to be independent on the boundary conditions.

As for the disordered FPU- $\beta$ lattice, it is observed that the value of $\beta$ depends on the boundary condition[13]. However, this result needs to be further checked as the authors in Ref.[13] used very short lattice, $N \leq 20$.

We should point out that the above results may not be very conclusive as we have an intermediate value of system size $N$. In order to get a more convincing conclusion, one needs to go 


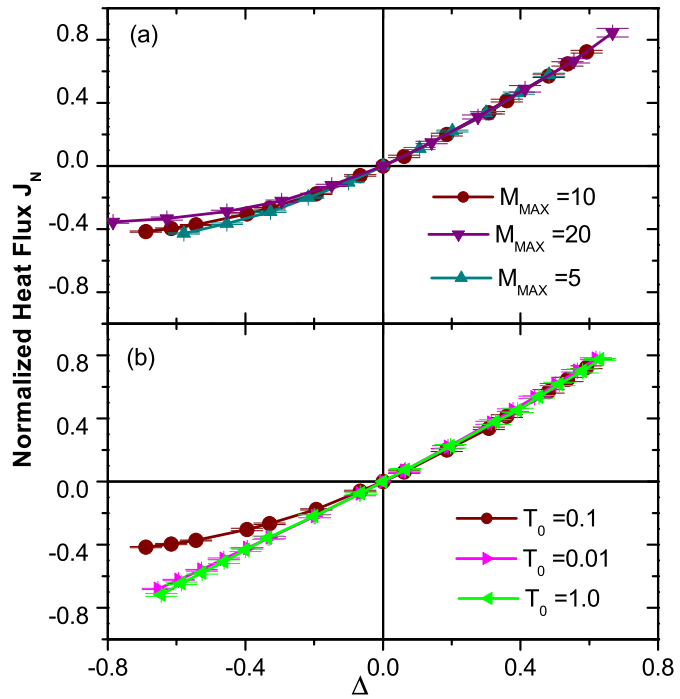

FIG. 3: (Color on-line) (a) Normalized Heat flux $J_{N}$ versus $\Delta$ for three different mass gradients. $N=200, T_{0}=0.1, M_{M I N}=1$ and $M_{M A X}=20,10$ and 5. $J_{N}=J / C$, where $C=0.0045,0.0080$, and 0.0145 for $M_{M A X}=20,10$ and 5 respectively. (b) Normalized Heat flux $J_{N}$ versus $\Delta$ for $T_{0}=1.0,0.1$, and 0.01. $N=200, M_{M I N}=$ $1, M_{M A X}=10 . J_{N}=J / C$, where $C=0.072,0.008$ and 0.0005 for $T_{0}=1.0,0.1$, and 0.01 respectively.

to large $\mathrm{N}$, say $N>10^{5}$, which is very difficult for current computer facilities. However, this is not the main purpose of the paper.

In the following, we study the thermal rectification in the mass graded anharmonic lattice. In order to avoid the effect of the Kapitza resistance at the boundary, we record the temperatures of the closest particles to bath particles at two ends, namely, the $3^{r d}$ and the $198^{\text {th }}$ for $N=200$ as $T_{l, r}$. For convenience, we set the temperature of heavy mass end $T_{l}=T_{0}(1+\Delta)$ and that of light mass end $T_{r}=T_{0}(1-\Delta)$, where $T_{0}$ is the average temperature of the system.

For comparison, we show the normalized heat flux $J_{N}(\equiv$ $J / C$ ) versus $\Delta$ in Fig $\sqrt{3}$ a) for three different mass gradients, where $C$ is a constant. When $\Delta>0$ for different mass gradients, the heat flux increases steeply with the increase of $\Delta$. However, in the case of $\Delta<0$, the heat flux changes a little when $\Delta$ changes. This asymmetry of heat flux with respect to $\Delta$ is called thermal rectification. We study the dependence of the rectification on the mass gradient (fix $M_{M I N}=1$ and change $M_{M A X}=20,10$ and 5). It is shown in Fig 3 a) that the larger the mass gradient, the more obvious the rectification.

To find the temperature dependence of rectification, we calculate normalized heat flux $J_{N}$ versus $\Delta$ for different temperature, $T_{0}=0.01,0.1$ and 1.0, (Fig $\left.3(b)\right)$. From this figure we can see that the thermal rectification vanishes in both high and low temperature limits. In the low temperature limit, the graded anharmonic lattice reduces to the graded harmonic lat-
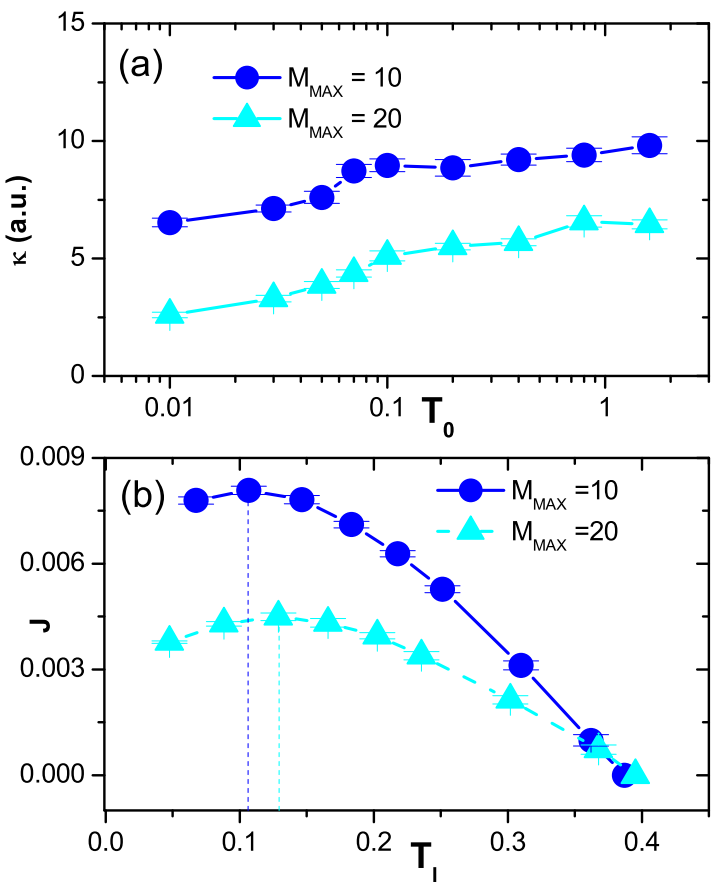

FIG. 4: (Color on-line) (a) Heat conductivity $\kappa$ versus temperature $T_{0} . \Delta=0.1$. (b) Heat flux $J$ versus $T_{l} . T_{r}$ is fixed at 0.4. $N=$ $200, M_{M I N}=1$ and $M_{M A X}=20$ and 10. The negative differential thermal resistances $(R<0)$ appears when $T_{l}<0.14$ and 0.11 for $M_{M A X}=20$ and 10 respectively.

tice in which no rectification exists. In the high temperature limit, the low frequency vibration modes, which dominate the heat conduction, in both ends can be excited, therefore no rectification is found either. In the case of $T_{0}=0.1$, the low temperature end is harmonic, while the high temperature end is strongly anharmonic, thus the vibrational spectra strongly mismatch with each other, which leads to the thermal rectification.

Finally, we should stress that the rectification is sensitive to boundary conditions. The effect can be observed only for fixed boundary conditions, because of the low frequency mode cannot be restrained for free boundary conditions.

We show temperature dependence of heat conductivity in Fig 4 (a) for the graded anharmonic lattice. Another important feature found in this 1D graded chain is the negative differential thermal resistance (NDTR)[2], namely, the larger the temperature difference the less the heat flux through the system. In order to illustrate the NDTR, we fix the temperature of the right (light mass) end at $T_{r}=0.4$ and plot the heat flux $J$ versus temperature $T_{l}$ in Fig 4 b). The differential thermal resistance is defined as $R=-\left(\partial J / \partial T_{l}\right)_{T_{r}=\text { Const }}^{-1}$. The NDTR is seen on the left part of the vertical line, where $J$ increases as $T_{l}$ is increased.

To understand the mechanism of rectification and NDTR, we calculate the power spectrum of particles close to the two ends, and then compare their spectrum with each other(Fig 5 ]. 


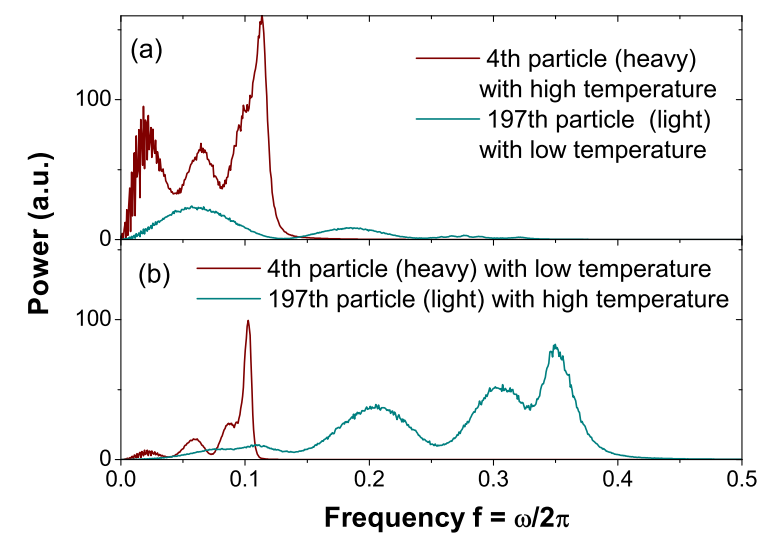

FIG. 5: (Color on-line) Spectra of the two end particles for system $N=200, T_{0}=0.1, M_{M I N}=1$ and $M_{M A X}=10$. (a) The heavy mass end (left) contact with high temperature bath $(\Delta=0.6)$ (b) The light mass end (right) contact with high temperature bath $(\Delta=-0.7)$.

When temperature is high enough, quartic term in FPU potential is the dominant term in the whole chain. So the coupling among modes is strong and low frequency modes, which contribute to the heat conduction most, are abundant in the spectrum. Then the flux depends mainly on temperature difference. So the quartic term is only dominant at the end with high temperature and the quadratic term plays the main role at the low temperature end, therefore, the low frequency modes cannot go through the system. The heat current is controlled by two competing effects: temperature gradients and overlaps of vibrational spectra. In the presence of mass gradient, there is a big difference between $\Delta>0$ and $\Delta<0$ in the spectra. As shown in Fig 5 a), where the left end (heavy mass) contacts with high temperature bath $(\Delta>0)$, the spectra of the two particles overlap in a wide range of frequencies, thus the heat can easily goes through the lattice along with the direction of temperature gradient. Whereas when the right end (light mass) contacts with high temperature bath $(\Delta<0$,Fig 5 (b) $)$, the spectra separate from with other. It can be seen that the light mass particle with high temperature oscillates mainly in high frequency, however the heavy mass particle with low temperature oscillates in low frequency. As a result, heat is difficult to go through the system although there is a temperature gradient. When $T_{0}$ goes to low temperature limit, the graded chain can be regard as a graded harmonic lattice whose thermal properties are like those of the homogenous harmonic lattice, then the flux depends only on the temperature difference.

In summary, we have studied thermal properties of 1D mass graded harmonic and anharmonic lattices in this paper. It is found that the temperature gradient can be built up in the 1D graded harmonic lattice chain due to the localization of high frequency gradon modes. The heat conductivity diverges with the system size linearly, $\kappa \propto N$. In the graded anharmonic lattices, the thermal conductivity diverges with system size as, $\kappa \sim N^{\alpha}$, the value of $\alpha$ seems to be independent of temperature and boundary condition.

The thermal rectification and the negative differential thermal resistance have been observed in the graded anharmonic lattice. This is quite similar to the recent nanotube experiment [3], in which half of the tube has been gradually mass-loaded on the surface with heavy molecules. Our results suggest that the graded materials might be used as thermal rectifier and thermal transistor.

This work is supported in part by an Academic Research Fund from Ministry of Education, Singapore, and the DSTA of Singapore under Project Agreement No. POD0410553.

* Electronic address: phylibw@ nus.edu.sg

[1] M. Terraneo, M. Peyrard, and G. Casati, Phys. Rev. Lett. 88, 094302 (2002); B. Li, L. Wang, and G. Casati, Phys. Rev. Lett. 93, 184301 (2004); B. Li, J. Lan, and L. Wang, Phys. Rev. Lett., 95, 104302(2005); J. Lan and B. Li, Phys. Rev. B 74, 214305 (2006); B. Hu, L. Yang, and Y. Zhang, Phys. Rev. Lett. 97, 124302 (2006); J. Lan and B. Li, Phys. Rev. B 75, 214302 (2007).

[2] B. Li, L. Wang, and G. Casati, Appl. Phys. Lett. 88, 143501 (2006).

[3] C. W. Chang, D. Okawa, A. Majumdar, and A. Zettl, SCIENCE 314, 1121 (2006).

[4] Y Koike, Graded index materials and components, in Polymers for lightwave and integrated optics, (ed. L. A. Hornak), Marcel Dekker, Inc., New Yor, p 71-104. 1992.

[5] J. P. Huang and K. W. Yu, Phys. Rep. 431, 87 (2006).

[6] G. L. Fischer et al, Phys. Rev. Lett. 74, 1871 (1995); R. S Bennink et al, Opt. Lett, 24, 1416 (1999); J. P. Huang and K. W. Yu, App. Phys. Lett. 85, 94 (2004); J.P. Huang and K. W. Yu, Oppt. Lett, 30, 275 (2005).

[7] J. J. Xiao, K. Yakubo, and K.W. Yu, Phys. Rev. B 73, 054201(2006).

[8] Z. Rieder, J. L. Lebowitz, and E. Lieb, J. Math. Phys. 8, 1073(1967).

[9] H Matsuda and K Ishii, Suppl. Prog. Theor. Phys. 45, 56 (1970).

[10] S. Lepri, R. Livi, and A. Politi, Phys. Rep. 377, 1 (2003) and the references therein.

[11] O. Narayan and S. Ramaswamy, Phys. Rev. Lett. 89, 200601 (2002).

[12] P. Grassberger, W. Nadler and L. Yang, Phys. Rev. Lett. 89,180601 (2002).

[13] H. Zhao, L. Yi, F. Liu, and B. Xu, Eur. Phys. J. B 54, 185 (2006). 